\title{
Modelling and Control of a Hydraulic Large Range Robot
}

\author{
U. Hirsch*; A. Jacubasch*; H.-B. Kuntze* \\ F. Eberle**; B. Göller** \\ * Fraunhofer-Institut für Informations- und Datenverarbeitung (IITB) \\ ** Institut für Reaktorentwicklung, Kernforschungszentrum Karlsruhe (KfK) \\ D-7500 Karlsruhe, Germany
}

\begin{abstract}
Large range robots are of increasing importance for various difficult applications at building sites or in hazardous areas. In collaboration with industrial partners the $\mathrm{KfK}$ has developed such extended multi joint robot (EMIR) which is driven by hydraulic actuators. The position control of EMIR is a tough problem due to the extreme nonlinearities of the kinematics and hydraulic actuators as well as the remarkable elasticities of the mechanics and hydraulics. In the first part of this paper a realistic physically transparent model of the robot will be presented. In the second part different suitable control concepts based on the model will be discussed.
\end{abstract}

Keywords: Hydraulic large range robot, automatic control, modelling of nonlinearities and elasticity, model-based control concepts

\section{MOTIVATION}

There are various important out-door applications in the fields of civil engineering, environmental technoloy or catastrophy management which require necessarily the introduction of a large range robot. Complying with the existing and expected demands of these important future-oriented markets in collaboration with different industrial partners $\mathrm{KfK}$ has developed the EMIR robot (Extended Multi Joint $\underline{R}$ obot) [1]. It is a six-joint machine powered by hydraulic servo drives. The mechanics of the EMIR is characterized by one vertical and five kinematically redundant horizontal joints which provide the robot with an amazing dexterity for complex manipulation tasks. At the TCP (Tool Center Point) the EMIR can carry payloads of up to $1500 \mathrm{~kg}$ within a working range of up to $22,5 \mathrm{~m}$. The hydraulic servo drives are cylinders for the five horizontal joints and one rotary actuator for the vertical base joint. The hydraulic oil flow of each actuator will be controlled by means of electro-magnetic (solenoid) servo valves. A safety logic valve between each hydraulic drive and servo valve checks permanetly the fluid pressure within the cylinder in order to prevent uncontrolled hazardous robot motions in the case of any hydraulic malfunctions (cf. Fig. 1). The control hardware is implemented on a conventional AT-386 computer including a signal processor board extension.

The static and dynamic system behaviour of the uncontrolled robot is characterized by several difficulties. Mainly due to the kinematics of the joints and the behaviour of the hydraulic actuators the response of the servo system is extremely nonlinear. The nonlinearity depends both on the actual joint position and the velocity. Especially the integrated safety logic block of the actuators seriously disturbs the system dynamics mainly in the case of lowering the TCP. An additional source of trouble for the control design is the spatially distributed elasticity of the slim and huge robot mechanics which is unfortunately superimposed by the elasticity of the hydraulic fluid within the actuators. Because of the highly nonlinear and elastic system behaviour of hydraulic actuators and robot mechanics for the closed-loop position control of the robot joints sophisticated model-based concepts are required.

In the first part of this paper a complex physically transparent robot model will be presented, which considers all nonlinear and elastic properties of hydraulics and mechanics. The model 
parameters are matched by means of experimentally obtained responses at the real EMIR. Based on representative simulations essential features of the uncontrolled system behaviour which are important for the control design are discussed. Based on the robot model in the second part of the paper different joint position control concepts will be presented and discussed with respect to the dynamic response and robustness. One of it is the model-based PFC-concept (Predictive Functional Control) which has been developed and successfully applied for industrial robots by the IITB [2]. It will be shown that PFC can also be applied to hydraulic large range robots like EMIR.

\section{MODELLING}

The difficult system behaviour of the robot is caused by the remarkable elasticities and nonlinearities both of the slim mechanics and of the hydraulic actuators. For a better transparency of the physical and mechanical behaviour in a first step the mechanics and hydraulic actuators are seperately discussed. The complete robot model will be obtained in a second step by integrating the partial models.

The results of modelling are

- a complex nonlinear robot model for simulation purposes and

- a simplified linear robot model of lower order for control design.

As simulation tools both the blockoriented system DISKOS [5] and the mechatronics simulation system ADAMS [6] are used. DISKOS (developed at the IITB) will be mainly applied for investigating hydraulic actuators and control algorithms while ADAMS will be prefered for the simulation of the complex robot mechanics and kinematics.

\subsection{Robot Mechanics}

The mechanics model is based on the simplified assumption that the EMIR consists of 24 main parts: 5 arms, 5 hydraulic cylinders, 5 pistons, 5 transfer levers, 4 thrust bars and 1 rotating joint (cf. Fig. 1, 3). The 5 arms are linked with each other by 5 transfer levers. The linear translational movement of the pistons against the cylinders $h_{i}$ will be transformed via transfer levers in a rotational movement of the arms $\alpha_{i}(i=1, \ldots, 5)$ according to the highly nonlinear kinematical realtions (cf. [3, 4] for details)

$$
\dot{\alpha}_{i}=\frac{\partial \alpha_{i}}{\partial h_{i}} \cdot \dot{h}_{i}=\ddot{u}_{i}\left(h_{i}\right) \cdot \dot{h}_{i}
$$

The 5 arms have a spatially distributed elasticity which has been approximated according to Fig. 2. Each arm is subdivided into 4 sections of almost equal elasticity. The sections correspond with rigid masses which are linked by damped springs. The masses are described by the matrices of inertia $\mathrm{J}_{\mathrm{i} 1}, \ldots, \mathrm{J}_{\mathrm{i} 4}$, the springs by the spring constant matrices $\mathrm{C}_{\mathrm{i} 12}, \ldots, \mathrm{C}_{\mathrm{i} 34}$ and the dampers by the damping constant matrices $\mathrm{K}_{\mathrm{i} 12}, \ldots, \mathrm{K}_{\mathrm{i} 34}$. Considering the distributed elasticity of the arms the complete mechanical model comprizes 39 rigid bodies with totally 96 degrees of freedom (DOF).

For the description and evaluation of the robot elasticity it is useful to distinguish between (cf. Fig. 3)

- stationary deflection caused by gravity and

- dynamic deflection caused by varying external forces (inertial forces, load changes).

If we consider for a better transparency only the small motion of joint 1 the dynamic deflection can be well described by the transfer function of the hydraulic piston stroke $h$ 


$$
\frac{\mathcal{L}\{h(t)\}}{\mathcal{L}\{F(t)\}}=G_{h}(s)=V_{h} \frac{b_{0}+b_{1} s+\cdots+b_{8} s^{8}}{a_{0}+a_{1} s+\cdots+a_{10} s^{10}}
$$

or the corresponding joint angle $\alpha_{1}$

$$
\frac{\mathscr{L}\left\{\alpha_{1}(t)\right\}}{\mathcal{L}\{\mathrm{F}(\mathrm{t})\}}=\mathrm{G}_{\alpha 1}(\mathrm{~s})=\ddot{\mathrm{u}}_{1} \cdot \mathrm{G}_{\mathrm{h}}(\mathrm{s})
$$

and of the tip angle $\alpha_{6}$

$$
\frac{\mathscr{d}\left\{\alpha_{6}(t)\right\}}{\mathscr{f}\{\mathrm{F}(\mathrm{t})\}}=\mathrm{G}_{\alpha 6}(\mathrm{~s})=\mathrm{V}_{\alpha 6} \frac{\mathrm{c}_{0}+\mathrm{c}_{1} \mathrm{~s}+\cdots+\mathrm{c}_{8} \mathrm{~s}^{8}}{\mathrm{a}_{0}+\mathrm{a}_{1} \mathrm{~s}+\cdots+\mathrm{a}_{10} \mathrm{~s}^{10}}
$$

with respect to the driving force $F$. For simplicity we denote $h_{1}=h$ and $F_{1}=F$. Of course the parameters of the linear relations (2), (3), (4) depend on the stationary robot position. The slowest (dominant) mode of the mechanics has a weakly damped frequency of about $5.4 \mathrm{~Hz}$.

\subsection{Hydraulic Actuators}

The components of the hydraulic actuators for the 5 horizontal robot joints are (cf. Fig. 4):

- a heavy duty servo drive (cylinder/piston)

- a proportional $4 / 3$-solenoid servo valve and

- a safety logic block.

By means of the 4/3-solenoid servo valve both the route and the intensity of the oil flow of the pump can be controlled. If a solenoid current $\mathrm{y}_{2}>0$ is chosen the valve guides the oil flow $\mathrm{Q}_{\mathrm{H}}$ into the lifting chamber of the cylinder. Simultanously the oil flowing out the lowering chamber $Q_{S}$ will be transported back into the tank. In the opposite case $y_{2}<0$ the lowering chamber will be filled and the lifting chamber will be emptied. If no current $\left(y_{2}=0\right)$ is applied both chambers remain closed.

The magnitude of the oil flow in the lifting side $Q_{H}$ and lowering side $Q_{S}$ depends on the flow resistance of each hydraulic component. Since turbulent conditions have to be assumed the pressure decay is proportional to the square of the flow $Q_{H}$ or $Q_{S}$ respectively. If in the considered actuator system 6 significant resistors are assumed which are located in the logic block, servo valve and the pump the stationary flow can be described by the nonlinear equations (1) ,..., (6) in Fig. 4. Thus $Q_{H}$ and $Q_{S}$ depend both on the constant parameters $k_{0}, k_{1}$ ,..., $\mathrm{k}_{60}, \mathrm{k}_{61}$ and of the variable parameters $\mathrm{y}_{2}, \mathrm{y}_{4}$.

By solving the nonlinear equations (cf. $[3,4]$ for details) the dependence $Q=Q\left(p_{0}, y_{2}\right)$ can be obtained. The corresponding diagram in Fig. 5 shows for normal pump pressure within the range 320 bar $<p_{0}<100$ bar an almost linear dependence between flow $Q$ and control current $y_{2}$. The linearization will be mainly caused by a self controlling pressure balance unit within the servo valve.

The aim of the safety logic block is to stop the oil to flow out of the lowering cylinder chamber as long as the pressure in the lifting chamber is under a safety threshold value. Thus, 
in the case of any brackage of the hydraulic vessels the robot mechanics loaded by gravity will maintain in a safe position.

The dynamics of the logic block is characterized by a very fast opening but a rather slow closing response (time constant $\sim 0,3 \mathrm{sec}$ ). The dynamics of the 4/3-solenoid servo valve is described by a bandwith of about $30 \mathrm{~Hz}$ in the slowest case $\left(y_{2}= \pm 100 \%\right)$. The solenoid servo valve has a static dead zone within the range $-10 \% \leq \mathrm{y}_{2} \leq 10 \%$.

The elasticity of the hydraulic actuator will be caused by the elasticity both of the oil and the oil vessels. If the elasticity will be approximately assumed to be concentrated within both cylinder chambers between pressure and flow the following "spring" equations are valid (cf. Fig. 4)

$\dot{\mathrm{p}}_{\mathrm{H}}=\left(\mathrm{Q}_{\mathbf{H}}-\dot{\mathrm{h}} \cdot \mathrm{A}_{\mathrm{H}}\right) \cdot \boldsymbol{\beta} / \mathrm{V}_{\mathbf{H}}$

$\dot{\mathrm{p}}_{\mathrm{S}}=\left(\mathrm{Q}_{\mathrm{S}}+\dot{\mathrm{h}} \cdot \mathrm{A}_{\mathrm{S}}\right) \cdot \boldsymbol{\beta} / \mathrm{V}_{\mathrm{S}}$

Obviously the chamber volumes $\mathrm{V}_{\mathrm{H}}, \mathrm{V}_{\mathrm{S}}$ depend on the piston stroke according to the equations

$\mathrm{V}_{\mathrm{H}}(\mathrm{h})=\mathrm{h} \cdot \mathrm{A}_{\mathrm{H}}+\mathrm{V}_{\mathrm{H} 0}$

$V_{S}(h)=V_{S 0}-A_{S} \cdot h$

In equations (4),...,(7) denote $A_{H}, A_{S}$ the effective piston areas (cf. Fig. 4) and $\beta$ the oil elasticity constant and $V_{H 0}$ und $V_{S 0}$ the initial volumes. If the robot mechanics is assumed to be rigid the joint 1 will oscillate wiht a weakly damped natural frequency of about $0.5 \mathrm{~Hz}$. Obviously the mechanics is much stiffer than the hydraulics.

\subsection{Integrated Model}

The integrated EMIR model will be obtained by linking the mechanics model and the actuator model via force and velocity according to the simplified block diagram in Fig. 6. In order to achieve a better transparency in the following only the motion of the main joint 1 will be considered. While some model parameters are exactly known (e. g. kinematics and geomtrics) other ones (e. g. the transfer functions $G_{h}(s), G_{\alpha 1}(s), G_{\alpha 6}(s)$ ) are less well known. They have to be determined experimentally.

The experimental system analysis comprized among other experiments [3] the stepwise opening of the proportional solenoid valve. Some of the step responses both for lifting and lowering of the stretched EMIR at a initial position of $\alpha_{1}(0)=45^{\circ}$ are shown in Fig. $7 \mathrm{a}$ and $7 \mathrm{~b}$. Considered will be the piston velocity $h$ for different step magnitudes $y_{2}$. It can be seen thàt the system behaviour is characterized both by weakly damped elasticities as well by strong nonlinearies. The TCP oscillates at different modes. The slowest (dominant) one has a natural frequency of about $0.7 \mathrm{~Hz}$. It can be seen that both during lifting and lowering the damping decreases significantly proportional to the step magnitude.

Obviously the nonlinear system behaviour is more disturbing in the lowering than in the lifting movement. That is mainly caused by the hydraulic safety block in interaction with the oscillating mechanics. The oscillations activate the oil pressure in the lifting chamber of the cylinder to oscillate, too. However, each cyclical pressure decay causes the safety block to interrupt temporarely the continous lowering movement.

As it has been shown by simulations and experiments the difficult dynamic system behaviour depends not only on the direction of motion but also on the actual joint position (Fig. 7). Since each joint position $\alpha_{1}$ corresponds kinematically with a certain location of the piston $h$ 
the oil elasticity $\beta / \mathrm{V}(\mathrm{h})$ can vary significantly due to variation of chamber volumes and the effective mass inertia (cf. [3] for details).

Based on the highly nonlinear simulation model roughly shown in Fig. 6 a simplified low order model for control design can be easily obtained by linearization. It has been found that a linear model of sixth or eigth order can provide a good tradeoff between accuracy and transparency.

\section{CONTROL CONCEPTS}

A control algorithm is required that provides a good dynamic tracking accuracy of the TCP position $r_{6}(t)$ and orientation $\left(x_{6}(t), y_{6}(t), z_{6}(t)\right)$ with respect to a desired cartesian trajectory which represents e.g. any out-door robot application. Tracking errors caused by acceleration or external load disturbances have to be compensated as completely as possible.

Of course the applied control concepts have to be based on the available sensors. For position measurement at this time each joint $1, \ldots, 5$ equipped with 12 bit joint angle resolvers which permit the direct measurement of $\alpha_{i}$ and $h_{i}$. Thus due to the elasticity of the mechanics the TCP position control has to be performed indirectly via the control of the joint positions $\alpha_{i}$ or piston positions $h_{i}$ respectively.

While this is the normal way of position control for industrial robots [10] which have a comparably stiff structure this indirect TCP control can be crucial for slim and elastic large range robots like EMIR. Thus, the introduction of a more subtile model-based control concepts using additional sensors seems to be useful. Both approaches will be discussed in the following.

\subsection{Force Feedback and Linearization}

As the step responses in Fig. 7a, b demonstrate the damping behaviour of the hydraulic actuators is rather weak. The stabilizing influence of dry (Coulomb-) friction can be almost neglected. According to the system structure diagram Fig. 6 a considerable improvement of the unsatisfactory dynamic system behaviour can be obtained by pressure or force feedback measures.

The most simple and wide spread concept of pressure feedback is based on the introduction of a bypass-like capillary line between both cylinder chambers $[7,8]$. It causes an artificial pressure dependent leakage flow $p_{H} \cdot k_{L H}$ and $p_{S} \cdot k_{L S}$ respectively which has a stabilizing influence (cf. Fig. 6). The main shortcomings of this artificial leakage concept are the increased oil consumption and the reduced safety of the robot. Without an additional electronic joint position control system the robot mechanics during standstill $\left(\mathrm{y}_{2}=0\right)$ slowly declines down due to gravity which is of course not acceptable.

More suitable for the considered robot control problem is a sensor-based force feedback concept which implies the measurement of the pressures $p_{H}$ and $p_{S}$ in both chambers of the hydraulic cylinder by means of highly linear transducers. This concept turned out to be very effective during position controlled experiments with EMIR [11]. In the feedback loop the effective driving force at the piston

$F=p_{H} \cdot A_{H}-p_{S} \cdot A_{S}$.

will be considered. In order the exclude the negative influence of external load disturbances onto the static accuracy and repeatability the force feedback signal $F$ will be filtered by a high pass with the transfer function 


$$
H(s)=k_{F} \cdot \frac{T_{H} s}{1+T_{H} s}
$$

The static nonlinearities caused by the hydraulic actuator (cf. Fig. 5) and the robot kinematics can be approximately compensated by a static prefilter which comprizes the inverted nonlinear analytical relations (cf. Fig. 8 and [1]).

\subsection{Conventional Position Control}

The applied conventional concept of joint position control being very similar to those ones of the most industrial robots [10] is characterized by a cascaded structure (cf. Fig. 8). The velocity control loop which corresponds for hydraulic robots with the above discussed force control loop (chapter 3.1 ) is superimposed by the position control loop. According to the colocated sensor (resolver-) location the piston stroke $h$ is the conrolled value.

The simple heuristic control law

$$
\dot{H}_{c}(s)=\dot{H}_{d}(s)+k_{p} \cdot\left[\frac{H_{d}(s)}{1+T s}-H(s)\right]
$$

provides both a P-feedback control of the position $h$ and a feedforward control of the velocity $h^{*}$ (cf. Fig. 8). The two control parameters $k_{p}$ (loop gain) and $T$ (low pass time constant) can be easily optimized according to straightforward heuristic rules without model knowledge. By means of the low pass term in the control law (11) discontinouities of the desired trajectories $h_{d}(t)$ exciting the elastic modes of the robot will be smoothed.

The tracking behaviour of the conventional control concept has been thoroughly investigated. It will be illustrated by the simulated time response of the piston velocity $\hat{h}(t)$ and the TCP deflection $\Delta r_{6}(t)$ shown in Fig. 9. Considered will be a short lifting and lowering of joint 1 around the initial position $\alpha_{1}(0)=45^{\circ}$ according to a desired velocity trajectory $h_{d}(t)$ which has a trapezoidal shape. A sampling rate of $\Delta t=32 \mathrm{~ms}$ will be assumed.

It can be seen that the piston velocity $\dot{h}(t)$ follows the desired velocity $h_{d}(t)$ with a phase shift of $\mathrm{T}=0,4 \mathrm{~s}$. Due to this control error and to the system elasticity the TCP has a dynamic deflection of about $\pm 17 \mathrm{~cm}$ around the static gravity deflection of about $18 \mathrm{~cm}$.

A higher dynamic accuracy of the piston movement $h(t)$ with respect to the desired trajectory $\mathfrak{h}_{d}(t)$ can be easily achieved by a further optimization of the parameters $k_{p}$ and $T$. However, the closer dynamic tracking of the piston causes more dynamic deflections of the TCP since the elastic robot modes will be more excited by the more discontinous trapezoidal velocity profile than by a smoothed continous one.

\subsection{Model Based Position Control}

A additonal significant inprovement of the dynamic TCP accuracy by means of the heuristic control algorithm (11) is not possible. It requires the introduction of a model-based control concept. One suitable approach is the Predictive Functional Control (PFC) concept which has been jointly developed by the IITB and the french research institute ADERSA for fast mechanical sytems and robot applications $[2,10]$.

The basic philosophy of PFC is quite straightforward and relies on the following 3 compo- $^{-}$ nents (Fig. 10).

(1) A dynamic internal model is used for the on-line simulation of the system response $\mathrm{x}_{\mathrm{M}}(\mathrm{i}+\mathrm{k})$ including disturbances $\mathrm{x}_{\mathrm{z}}(\mathrm{i}+\mathrm{k})$. 
(2) A reference trajectory $\mathrm{x}_{\mathrm{r}}(\mathrm{i}+\mathrm{k})$ traces out a smooth transition from the actual process output $x(i)$ (e. g. joint position $q_{i}$ ) to the desired trajectory $x_{d}(i+k)$ within a chosen

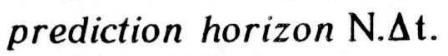

(3) An optimal control $u^{*}(i+k)$ forces the predicted process output $x_{p}(i+k)=x_{M}(i+k)+$ $x_{z}(i+k)$ as closely as possible to the reference trajectory.

Assuming a linear internal model and a first order reference trajectory the resulting control law for each joint is described by the linear equation

$u(i+1)=u(i)+k^{T} x_{r}(i)+a^{T} x_{M}(i)+b^{T} u(i)+h^{T}\left(x(i)-x_{p}(i)\right)$

where the constant control parameters are included in the vectors $\mathbf{k}, \mathbf{a}, \mathbf{b}$ and $\mathbf{h}$. The predicted response of $x_{r}, x_{M}, u$ and the past response of $x, x_{p}$ in few significant point is represented by the corresponding vectors $x_{r}, x_{M}, u, x, x_{p}$ (cf. [2] for details).

As regards both memory and sampling rate the requirements of the PFC algorithm (11) are very moderate. Applying an integrated $\mathrm{PC}$-assisted design technique including experimental model identification and succeeding PFC parameter calculation the implementation costs remain rather low.

PFC has been applied to the joint position control of the EMIR. Assuming again a sampling rate of $\Delta t=32 \mathrm{~ms}$, a time horizon of $\mathrm{N} \cdot \Delta t=20 \cdot 32 \mathrm{~ms}=640 \mathrm{~ms}$ (cf. Fig. 10) and a linear internal model of sixth order the tracking response shown in Fig. 11 will result. The boundary condtions of the example are the same as in Fig. 9. It can be seen that the shape of the velocity profile $h(t)$ is better smoothed than for the conventional control. Therefore the dynamic TCP deflection $\Delta r_{6}(t)$ will be smaller.

A further improvement of the dynamic performance can be achieved if PFC is directly applied to the TCP angle $\alpha_{6}$. This angle can be measured by means of the joint resolvers and an additional deflection sensor (e. g. strain gages). Assuming a linear internal model of eighth order and the same boundary conditions as in Fig. 11 the tracking behaviour shown in Fig. 12 will result. It can be seen that the dynamic TCP deflection is much smaller during the lifting and stand still phase. The deterioration during the lowering phase is mainly due to still existing internal model mismatch.

\section{CONCLUSIONS}

The dynamic system behaviour of hydraulic large range robots is very difficult due to extreme nonlinearities and elasticities of mechanics and hydraulic actuators. According to these difficulties a position control of high dynamic performance demands necessarily the introduction of model-based control concepts. In this paper diffferent model-based concepts which can solve the tough control problem will be presented.

\section{REFERENCES}

[1] Blume, Chr.; Gremminger, U.; Messemer, G.; Smidt, D.; Wadle, M.: "EMIR - a combination of manipulator and robot for new out-door applications in unstructured environments". Proc. 1989 IEEE Int. Conf. on Robotics and Automation, May 14-19, 1989, Scottsdale/Arizona, pp. 383-390.

[2] Kuntze, H.-B.; Jacubasch, A.; Hirsch, U.; Richalet, J.; Arber, Chr.: "On the application of a new method for fast and robust position control of industrial robots". Proc. 1988 IEEE Int. Conf. on Robotics and Automation, April 24-29, 1988, Philadelphia, pp. 1574-1580. 
[3] Hirsch, U.; Jacubasch, A.; Kuntze, H.-B.: "Modellbildung und Regelung des Großroboters EMIR". Abschlußbericht Nr. 10247 (A 27000) des IITB für die KfK/IFR, October 8, 1990.

[4] Göller, B.; Messemer, G.: "Das hydraulische Verhalten des EMIR - Messungen und Rechnungen für das Gelenk 1". Primärbericht der KfK/IRE (17.01.04P05A), Karlsruhe, Januar 1990.

[5] DISKOS-Benutzerhandbuch, Version PC unter MS-DOS, IITB-Bericht Nr. 9982, Karlsruhe, 03.11.1986.

[6] ADAMS User's Manual, Version 5.2, Mechanical Dynamics Inc. (MDI), Ber. MDI 01001-02, Ann Arbour, MI 48105-3203, October 1987.

[7] Guillon, M.: "Hydraulische Regelkreise und Servosteuerungen". Carl Hanser-Verlag, München, 1968.

[8] Skoff, S.; et. al.: Fluid power and controlled ideas from robotic designs". Hydraulics \& Pneumatics (1989), May, pp. 49-61.

[9] Tuncelly, A.C.; Günes, H.; Longchamp, R.: "Hydraulic axis control using pressure feedback". Proc. IEEE Int. Workshop on Intelligent Motion Control, Istanbul (1990), August, 20-22, pp. 663-668.

[10] Kuntze, H.-B.: "Position control of industrial robots - impacts, concepts and results". Proc. 2nd IFAC Symp. on Robot Control SYROCO'88, Karlsruhe (FRG), Oct. 5-7, 1988.

[11] Eberle, F.; Lang, G.; Scharnowell, R.; Wadle, M.: "Control experiments for concrete surface removal with a large range redundant manipulator". Proc. IEEE Int. Workshop on Advanced Motion Control, Keio University Yokohama (Japan), March 29-31, 1990.

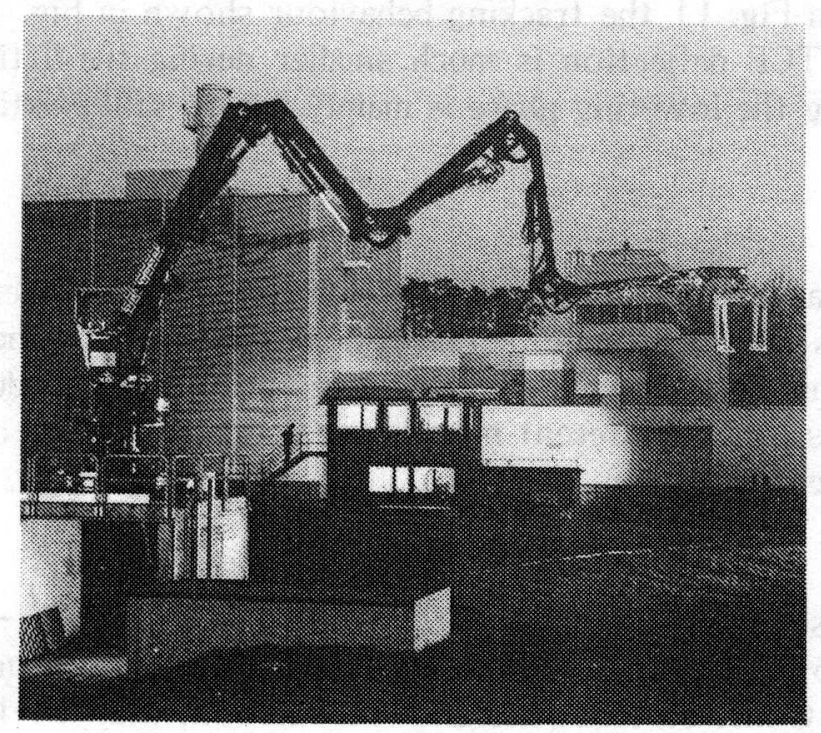

Fig. 1: Extended Muiti-joint $\underline{\text { Robot }}$ (EMIR) at the $\mathrm{KfK}$

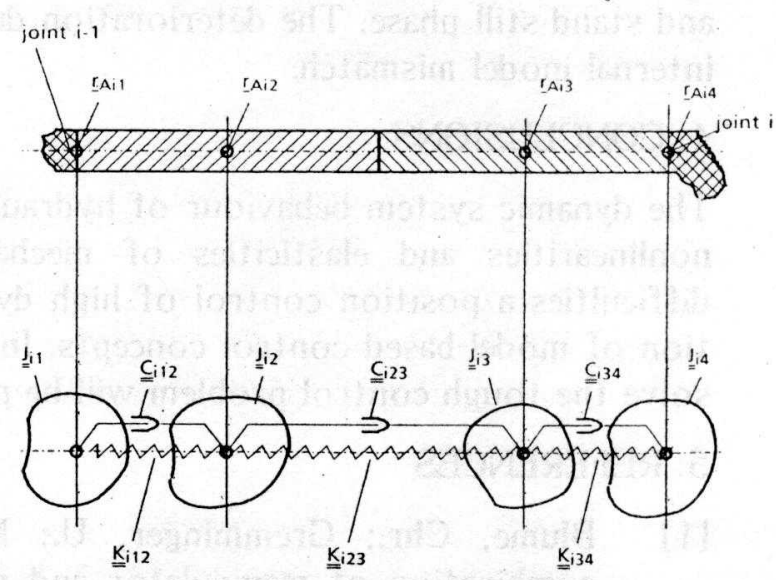

Fig. 2: Approximation of the spatially distributed arm elasticity by a mass-spring approach 


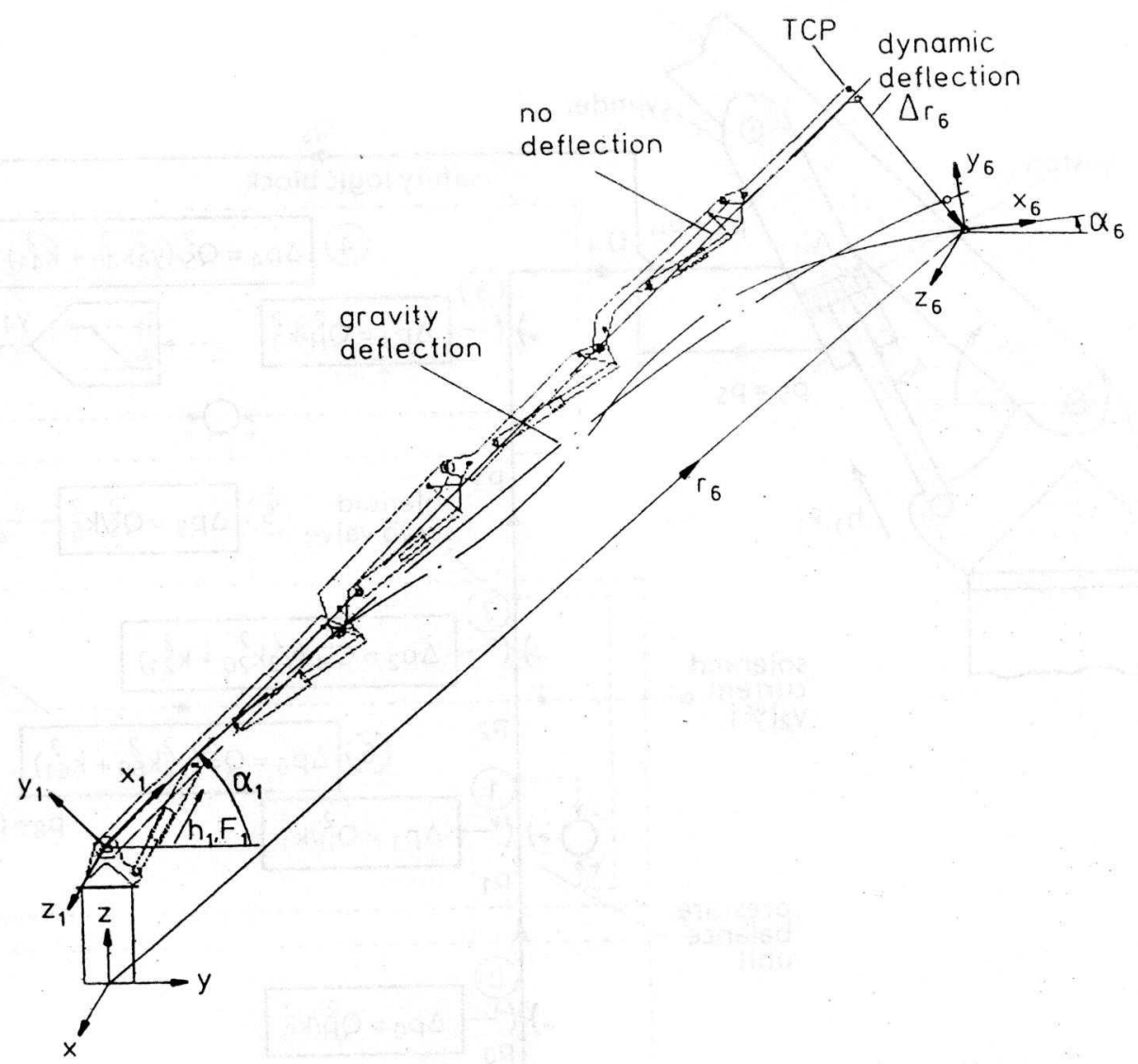

Fig. 3: Stationary and dynamic deflection of the stretched $\operatorname{EMIR}\left(\alpha_{1}=45^{\circ}\right)$

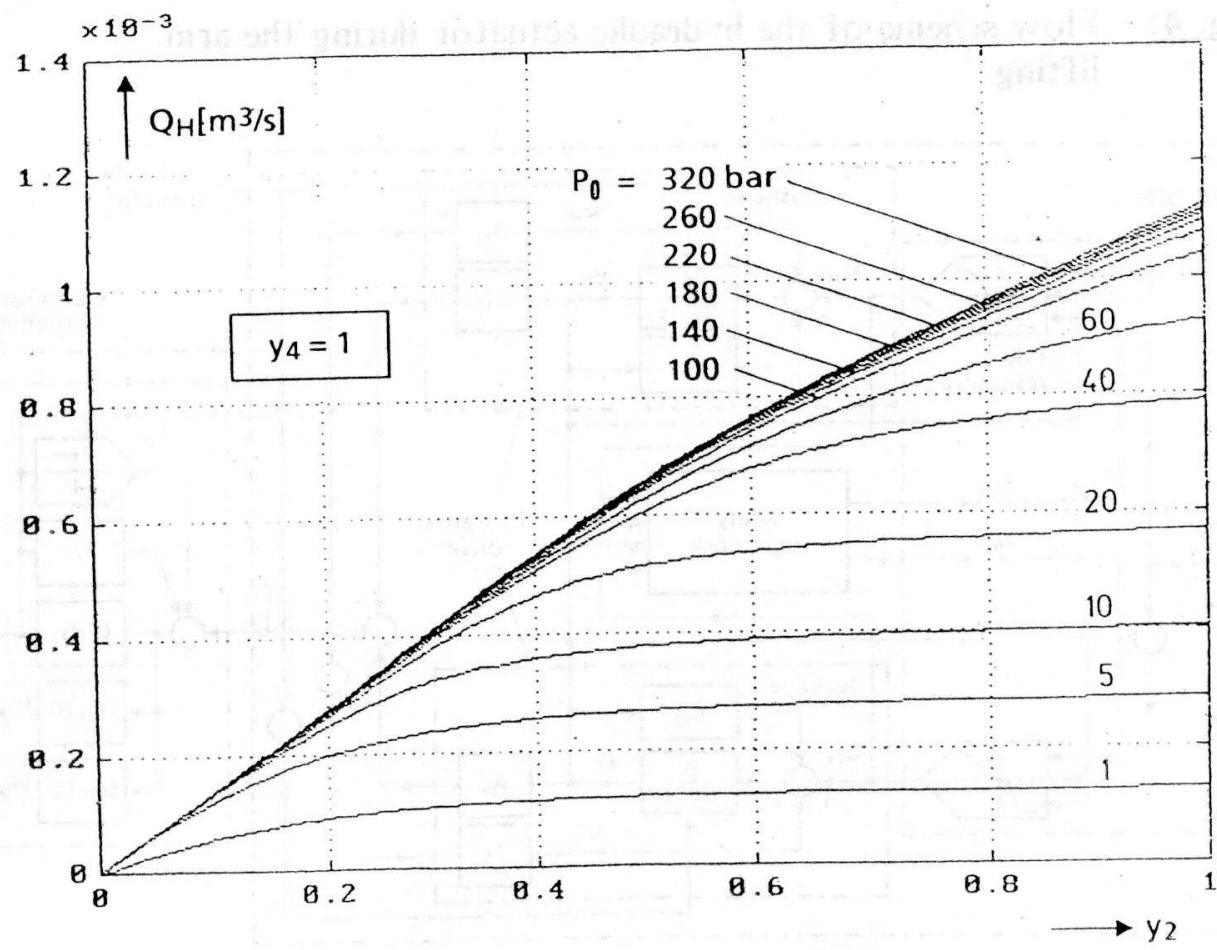

Fig. 5: Hydraulic oil flow $Q_{H}$ as function of the solenoid current $\mathrm{y}_{2}$ during arm lifting 


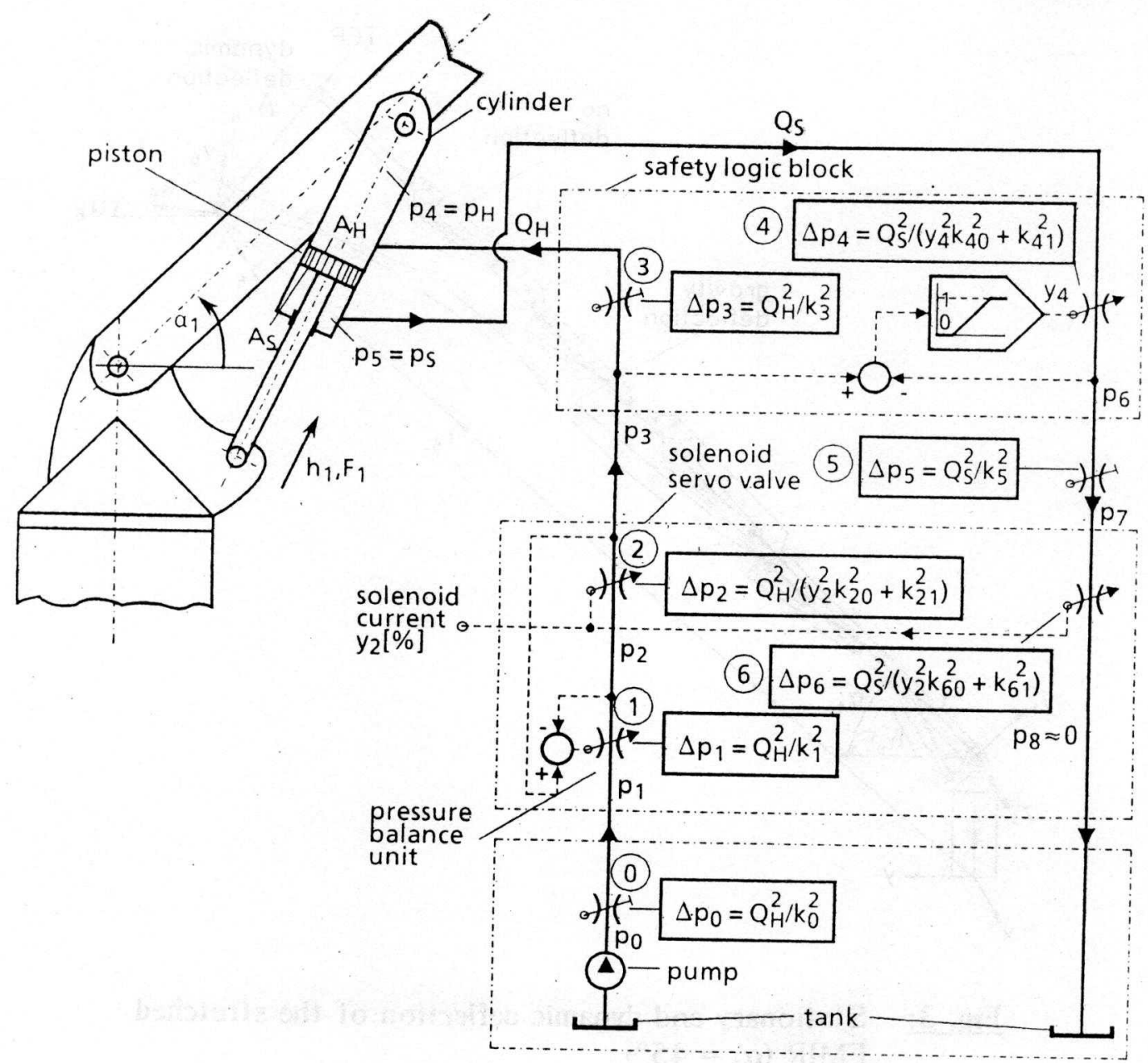

Fig. 4: Flow scheme of the hydraulic actuator during the arm lifting

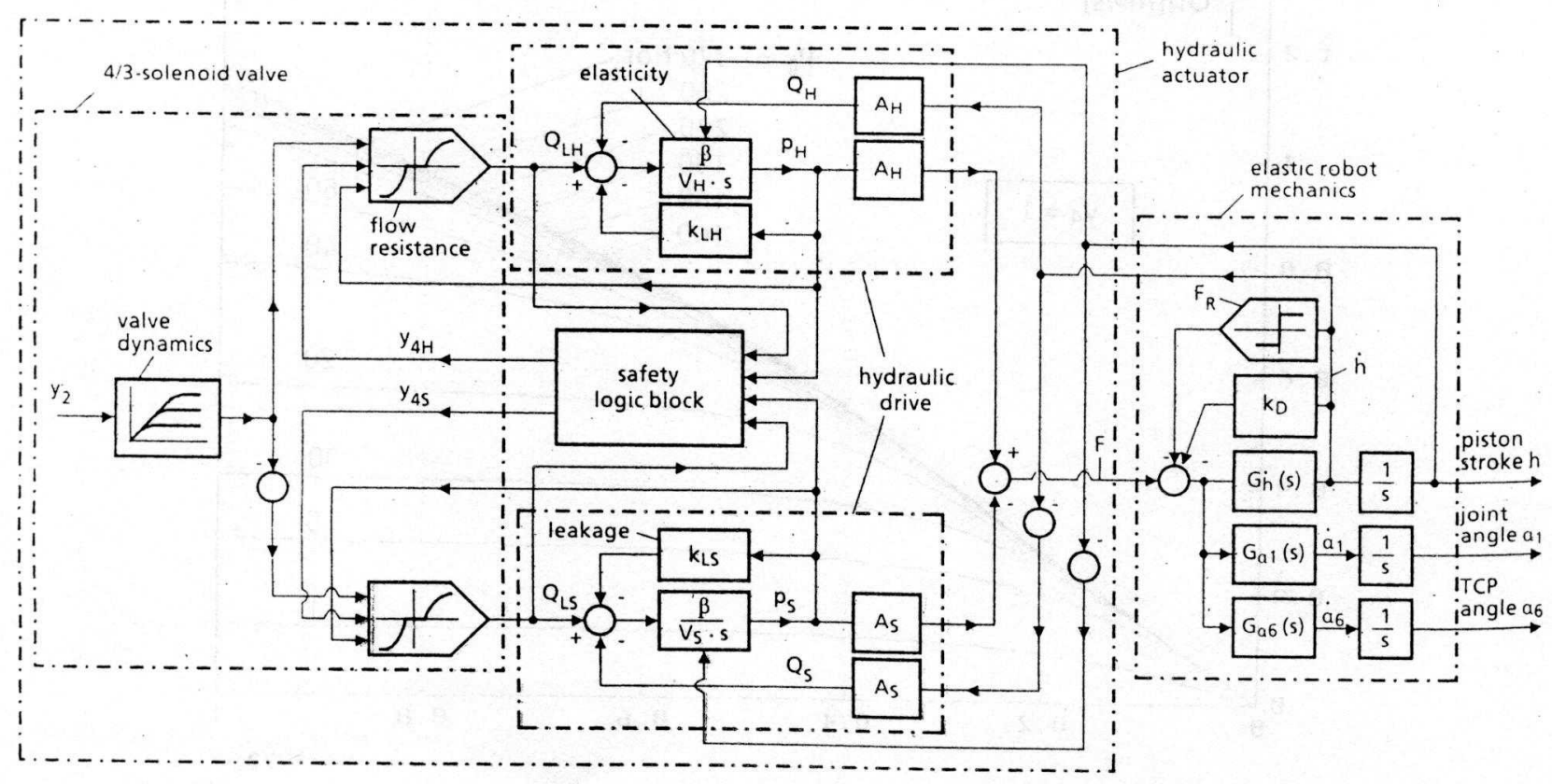

Fig. 6: Block diagram of the DISKOS simulation model 

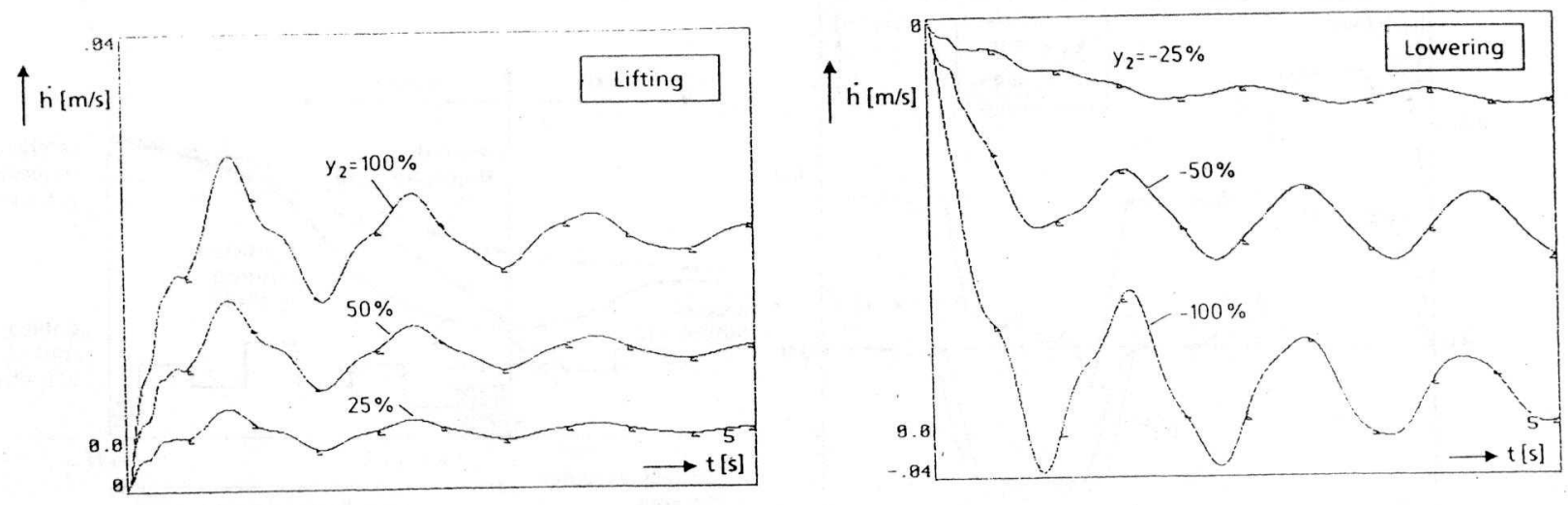

Fig. 7: Step response of the TCP angle velocity $\alpha_{6}$ in the lifting case (a) and lowering case (b)

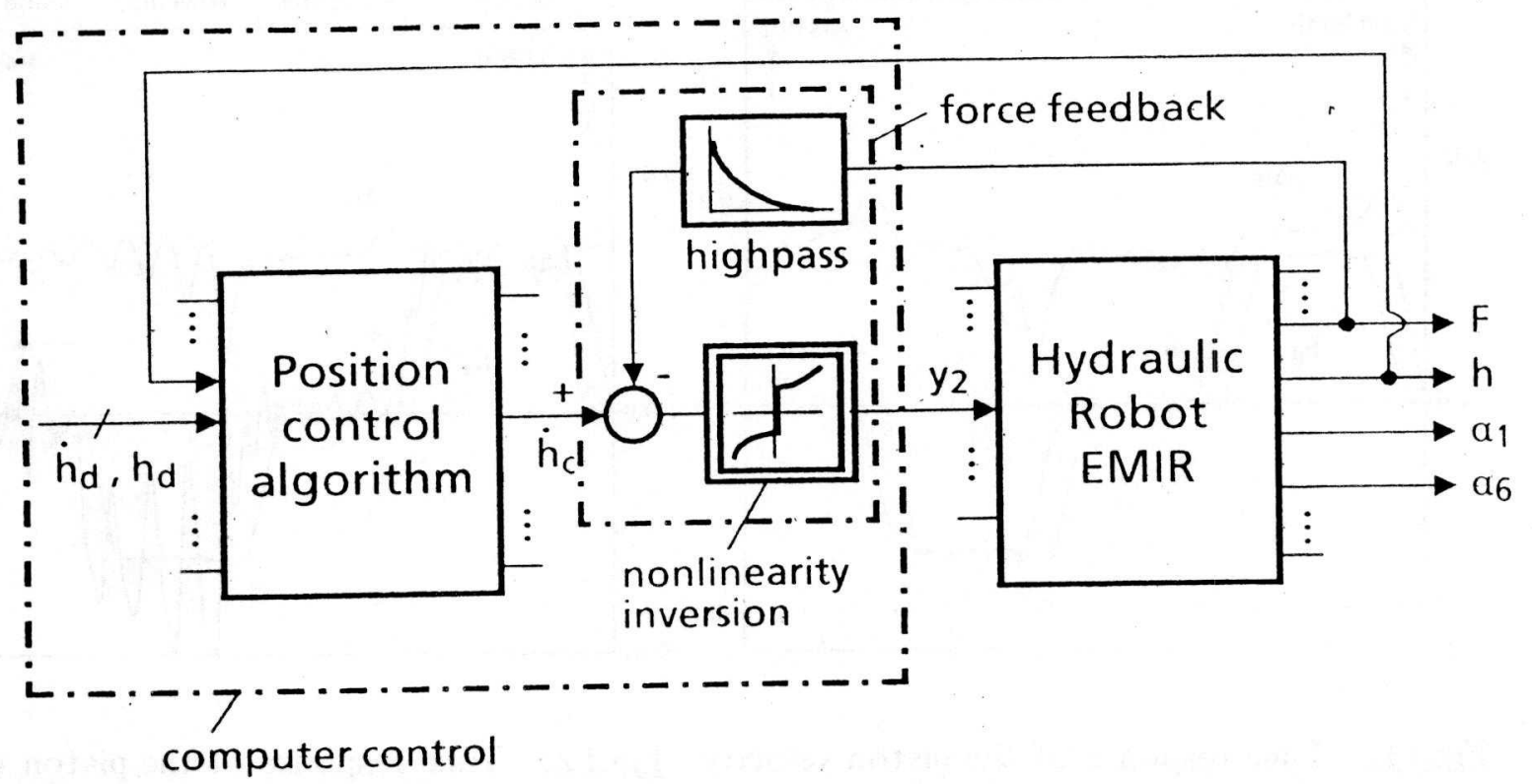

Fig. 8: Principle of force feedback control superimposed by position control 


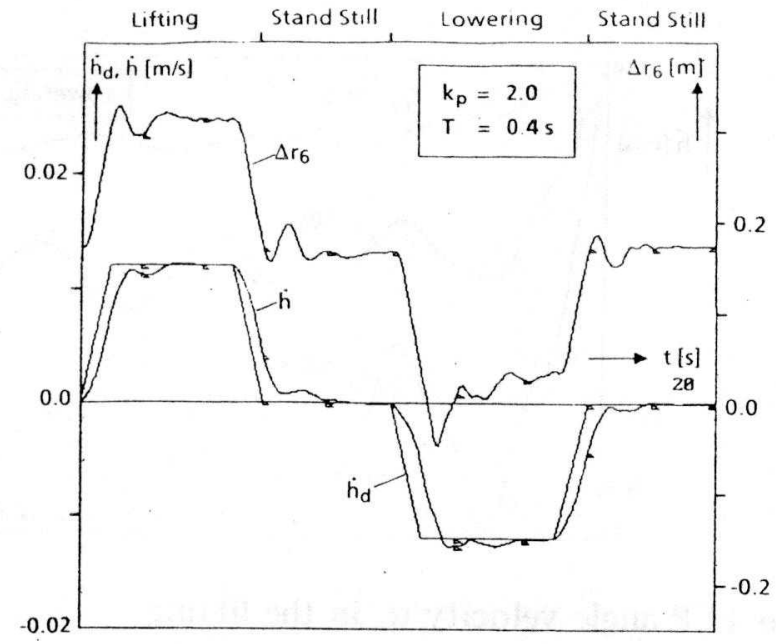

Fig. 9: Time response of the pist on velocity $h$ and the TCP deflection $\Delta r_{6}$ for the conventional joint control

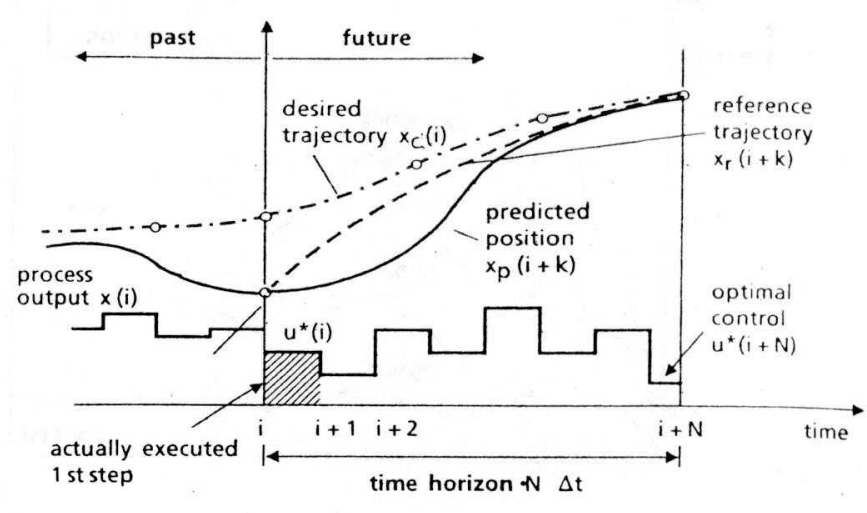

Fig.10: Principle of predictive functional control (PFC)

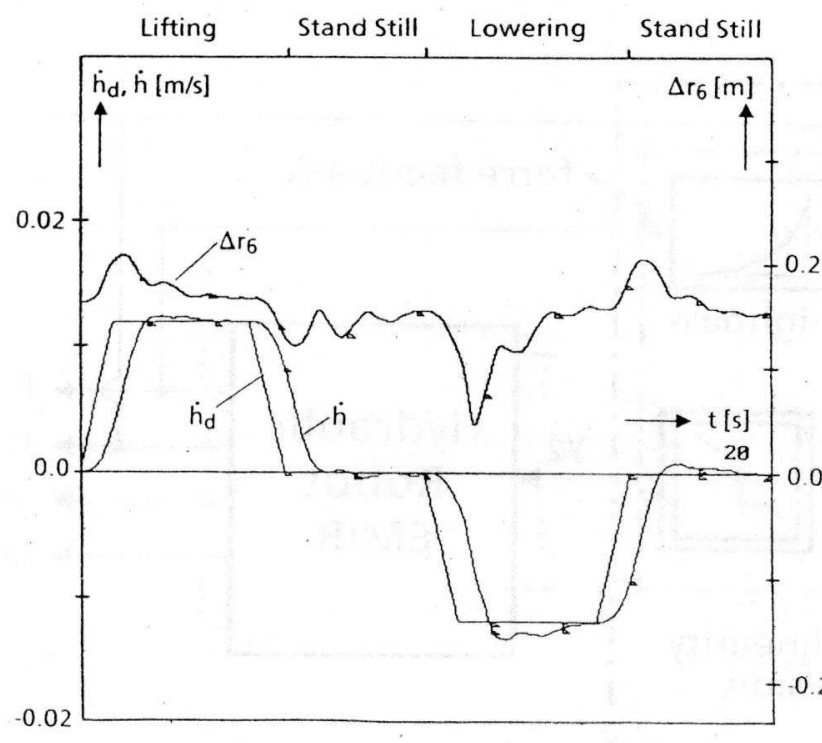

Fig.11: Time response of the piston velocity $h$ and the TCP deflection $\Delta r_{6}$ for PFC-joint control

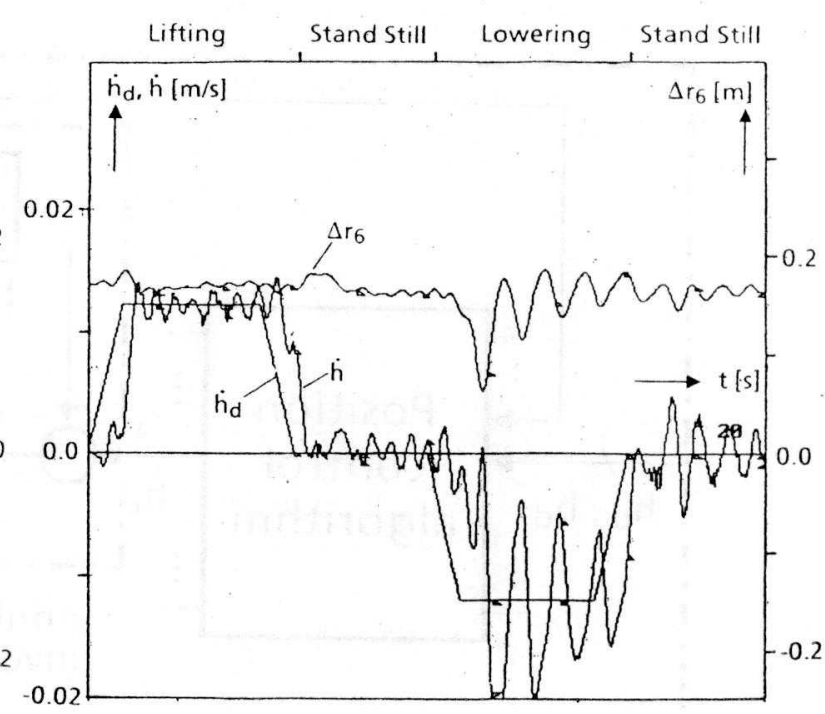

Fig.12: Time response of the piston velocity $h$ and the TCP deflection $\Delta r_{6}$ for PFC endpoint control 